\title{
Study of Position-Based Routing Protocols in Vehicular Ad Hoc Networks
}

\author{
Guanqi Wen ${ }^{1, a}$, Zhong Wang ${ }^{1, b}$, Shaolei Zhang ${ }^{1, c}$, Jing Wang ${ }^{1, d}$ \\ ${ }^{1}$ Computer Staff Room, Xi'an Research Institute of High Technology, Xi'an, 721006 \\ a email, ${ }^{b}$ email, ${ }^{c}$ email, ${ }^{d}$ email
}

Keywords: Position-based Routing Protocols, Mountain Environment, Vehicular Ad Hoc

\begin{abstract}
In high-speed driving, vehicles can communicate with each other or with roadside infrastructure and direct communication times, resulting in the existing road network dynamically and rapidly build a self-organizing, distributed control vehicle communication network. In the mountain environment, due to the road bumps, complicated surrounding environment, communication conditions often vary widely. This paper studies the characteristics of sub-mountain-vehicle ad hoc network, analyzes the content of automotive MANET routing protocol, we proposed some improvement ideas. I believe this research has a certain reference value for the vehicle-mounted ad hoc network technology under complicated natural environmental conditions.
\end{abstract}

\section{Introduction}

For the mountain transport network, the important environmental factors are the terrain, mountain disasters, but mountain terrain and disaster closely related to the topography, geology, precipitation, vegetation and other environmental factors. Therefore, a comprehensive analysis of the geographical environment of mountain transport network is actually a comprehensive analysis of topography, geology, vegetation and other environmental factors, such analysis of the analysis and evaluation of the presence of mountain transport network prediction and prevention of disasters and the environment has important significance. For example, along the hydrology, climate, topography and other layers of the multi-layer stack traffic lines and it can obtain basic natural conditions of the line; superimposed along the engineering geology, environmental geology layers transit line, available construction traffic conditions along and other information. From a technical perspective implemented method, appropriate scale to meet the analytical needs of various environmental factors the same pattern projection and attribute data storage is a prerequisite, technical analysis, including graphics mountain transport more environmental factors superimposed to generate in new thematic maps and multi-factor analysis to obtain the relationship between quantitative characteristics calculated in accordance with the contents of a certain mathematical model.

\section{Research on Routing Based on Topology}

Ad hoc network is a wireless distributed architecture and the emphasis is multi-hop, self-organization, no central concept. So you can put vehicular ad hoc defined as a fast-moving outdoor communication network, it was also called vehicular ad hoc self-organizing traffic information system. Vehicular ad hoc basic idea is that within a communication range of the vehicle can exchange information and data onboard sensor detect the respective vehicle speed, location, etc. to each other and automatically connect to establish a mobile network. Single-hop communication range of the node only a few hundred meters to one thousand meters, each node (vehicle) is not only a transceiver, is also a router, so a multi-hop manner forwards the data to further vehicles.

Routing protocols are VANET study an indispensable link, in particular due to the frequent changes in network topology VANET node moving fast, routing technology has become one of the major challenges VANET.

Network Routing basically topology-based routing protocol, nodes in the network is routed through all the other nodes periodically exchange routing information. According to different ways 
of routing establishment on MANET routing protocols can be divided Proactive routing protocols, on-demand routing protocol and hybrid routing protocols. Proactive routing protocols, also known as table-driven routing protocol in this protocol, whether or not there is communication requirements, each node periodically broadcast routing packets to exchange routing information, maintains a reach other nodes contain routing information table. Once the source node to send data packets, you can get a pointer to the destination node routing immediately typical routing table represented DSDV protocol.

Demand routing protocol is a self-routing protocol type specific networking, it can reduce the routing overhead and improve network throughput. Demand routing protocols including routing discovery and routing maintenance of the two processes. When a route request packet reaches the destination node, the destination node will be the source node sends a route request response packet. Thus, between the source and destination nodes will establish a bi-directional activation pathway. Activation pathway is defined as the time requiring of establish route setup delay. With the topology changes when a certain link activation path is interrupted, routing maintenance process is started. Demand routing protocols include DSR, AODV, etc.

Hybrid Routing Protocol is a protocol routing table and on-demand routing protocols combined. It uses the routing protocol table in a small area local area, local area to find the route to the nodes of the on-demand routing protocols. This link will be limited to changes in the local area.

\section{The Position-Based Routing}

Position-based routing requirements of each node in the network can be equipped with a dynamic of their own location and movement information means precise positioning and measurements (such as GPS), and then use the information obtained for routing and data transmission. Location routed through the introduction of geographic information nodes, topology routing algorithm reduces the number of deficiencies. Typically, each node by using GPS or other similar pointing device to get your location information, the network each node only needs to know the location information the communication within a radius of neighbors, the routing through the establishment of only a few single-hop topology information can be completed. Therefore, the data transmission source node to the destination node only needs to know the location and purpose of each data node forwarding nowadays hop node location can be achieved without the need for additional topology information. This kind of position-based routing does not require the establishment and maintenance of routing nodes do not store routing information table in the entire data transmission, it does not need to send routing update routing information. Therefore, this type of routing protocol can better adapt to changes in network size and topology. Position-based routing protocol mainly includes two aspects: (l) location service, figuring out how to get your own node location, location, location and purpose of the problem neighbor nodes; (2) packet forwarding strategy to solve the problem of the next hop. Currently proposed several different position-based routing protocols for inter-vehicle communications protocol is the main representative GPSR.

\section{A Location Protocol and Its Improvement Research}

Herein GPSR protocol module uses a GLS position-based service and a combination of greed and border forwarding strategy is similar. GPSR include the following elements:

(1) Hello packet via a node periodically broadcasts Hello messages to maintain data list its neighbor nodes. It can send configuration parameters to change the interval between Hello messages.

(2) Holding the local node connectivity within a node if more than a specified period of not Neighbor Expiry Time Hello message received from a neighbor node, it is assumed that node access route has been lost.

(3) Initial flooding to start node in the network, all nodes to initiate a comprehensive flooding in the network. While the number of initial flooding depends on each node sends a Number of Initial Floods attribute specified. 
(4) The position of the node assumes that each node can determine its position by the GPS. The location of the destination node is determined by flooding the network position information of other nodes.

(5) Location Update. When a mobile node is beyond a specified distance, it sends a flood it with new position information packet to inform other nodes. This flooding information is also sent when a node boundary crossing signal area.

(6) Fuzzy routing. In order to reduce the load generated by the flooding update, the flooding scope is limited, fuzzy route only those who need to know this change will be a node receives this flooding information.

(7) Neighborhoods quadrants. In order to optimize the flooding, the entire network is divided into multiple neighborhoods and the size of the adjacent area can be specified by the global attributes. These regional organizations are through a method of classifying them and each advanced region is divided into four lower regions.

GPSR and AODV are under OPNET simulation results, including the packet arrival rate and the average number of hops. It can be seen: GPSR protocol packet arrival rate was significantly better than AODV protocol. This is due to the AODV protocol-by-hop routing, we need to maintain the routing table, although AODV contains the destination node sequence number in the routing entry, but due to strenuous exercise node makes topology changes frequently, thus increasing the number expired routing entries expire the route will affect the accuracy of the route, do not apply to mobile network topology changes frequently. The GPSR greedy forwarding strategy does not need to maintain a complete routing, nor use the routing table and routing cache and intermediate forwarding node only needs to know the location information of the neighbor node and the destination node and forwarding policy decisions. The figure dropped packet arrival rate is primarily a destination node topology accuracy and fragmentation caused. Second, because AODV protocol-by-hop routing and GPSR taken greedy forwarding, smaller number of end-average hop GPSR. Therefore it concludes: rapid changes in the topology of the network, GPSR more suitable for VANET.

OPNET GPSR data in the header portion includes the address of the destination node, the location information, the shortest distance from the destination node in the current state, including survival and the recording process through packet flow forwarding node. In EGPSR protocol header added new secondary transfer destination node location information for the current state of the secondary transfer destination node and a second shortest distance forwarding flag. The control information is added to increase the share control information packet structure redundancy proportion. However, by increasing the control information it is able to make the routing policy to increase the packet arrival rate in the urban scene. The simulation results show EGPSR packet arrival rate is significantly higher than GPSR.

VANET node IP module into a TTL counter in the IP header of each packet, when each application layer to send a new packet for TTL initialize a fixed value, whenever a packet is successfully forwarded to the next hop node TTL- 1 . When the TTL equals 0 , the packet will be discarded. Because the packet forwarding process GPSR excessive or misguided boundary nodes forwarding hops excessive, leading to forwarding failure.

\section{Conclusion}

Although this paper makes some preliminary research on VANET position-routing protocol proposes some improvement ideas, but there are many aspects which need further discussion, for example, in the OPNET simulation scenario file can be added to the terrain more realistic simulation scenarios described. In order to obtain the appropriate terrain, it needs for geographic mapping software in-depth research and if, in order to describe the real traffic conditions, require in-depth study of microscopic traffic simulation software to enable OPNET, geography software, traffic simulation tripartite combination. Due to limitations of simulation conditions, the number of nodes in this paper is relatively small, the smaller the size of the network, thus further increasing the size of the simulation in the follow-up study in order to more realistically simulate real-world 
scenarios.

\section{References}

[1] BKarp, H T Kung. GPSR: Greedy perimeter stateless routing for wireless networks [J]. Proceedings of the 6th annual international conference on Mobile computing and networking, 2000, 1 (1): 243-254.

[2] CLochert, H Hartenstein. A routing strategy for vehicular ad hoc networks in city environments [A]. Proceedings of IEEE Intelligent Vehicles Symposium (2003) [C]. NY: IEEE, 2003. 156-161.

[3] C Lochert, M Mauve. Geographic routing in city scenarios [J]. ACM SIGMOBILE Mobile Computing and Communications Review (MC2R), 2005, 9 (1): 69-72.

[4] Yang Xia, Chai Kiat Yeo, Bu Sung Lee. Hierarchical Cluster Based Routing for Highly Mobile Heterogeneous MANET. Communications and Information Technologies: International Symposium [C]. NY: IEEE, 2007.936-941.

[5] R A Santas, R M Edwards, A Edwards, D Belis. A novel cluster-based position-routing algorithm for inter- vehicular communication. Indoor and Mobile Radio Communications, IEEE proceedings of the 15th Annual Symposium [C]. NY: IEEE, 2004. 1032-1036. 